\title{
OPEN M1-like macrophage polarization prevails in young children with classic Hodgkin Lymphoma from Argentina
}

Received: 5 November 2018

Accepted: 8 August 2019

Published online: 03 September 2019

\author{
O. Jimenez ${ }^{1}$, M. H. Barros ${ }^{2}$, E. De Matteo ${ }^{1}$, M. Garcia Lombardi ${ }^{3}$, M. V. Preciado ${ }^{1}$, \\ G. Niedobitek ${ }^{2}$ \& P. Chabay ${ }^{1}{ }^{1}$
}

The microenvironment in classical Hodgkin lymphoma (cHL) comprises a mixture of different types of cells, which are responsible for lymphoma pathogenesis and progression. Even though microenvironment composition in adult cHL has been largely studied, only few groups studied pediatric $\mathrm{cHL}$, in which both Epstein Barr virus (EBV) infection and age may display a role in their pathogenesis. Furthermore, our group described that EBV is significantly associated with cHL in Argentina in patients under the age of 10 years old. For that reason, our aim was to describe the microenvironment composition in 46 pediatric cHL patients. M1-like polarization status prevailed in the whole series independently of EBV association. On the other hand, in children older than 10 years, a tolerogenic environment illustrated by higher FOXP3 expression was proved, accompanied by a macrophage polarization status towards M2. In contrast, in children younger than 10 years, M1-like was prevalent, along with an increase in cytotoxic GrB + cells. This study supports the notion that pediatric cHL exhibits a particular tumor microenvironment composition.

The interaction between tumor and its microenvironment has been a key topic in cancer research for the last years. cHL has a unique morphological presentation with a minority of neoplastic cells, and a large majority of non-malignant reactive immune cells ${ }^{1,2}$. This extensive milieu of non-malignant cells is recruited by Hodgkin Reed-Sternberg (HRS) tumor cells and comprises B and T cells, together with eosinophils, neutrophils, mast cells, fibroblasts and macrophages ${ }^{1-3}$. HRS cells secrete cytokines, chemokines, and other immunomodulatory factors, such as IL-10, CCL17, galectin-1, and indoleamine 2,3-dioxygenase (IDO) that both recruit Th2 and regulatory FOXP3 + CD4+ T cells (Treg), favoring the differentiation of tumor-infiltrating T cells into regulatory and Th2 cells. HRS cells also secrete macrophage migration inhibition factor, which induces macrophage M2 polarization, also rendering a tumor microenvironment favorable for tumor cell growth and survival ${ }^{4}$.

Most studies about tumor microenvironment composition in cHL were performed in adults and a minority in pediatric cases. Moreover, these pediatric studies still wait for further validation ${ }^{5-7}$. This is a crucial point since the immune response against HRS cells and consequently the tumor microenvironment composition may differ between adult and children ${ }^{6,8,9}$. For instance, EBV + pediatric $\mathrm{cHL}$ cases display predominantly GrB + cytotoxic/Th1 microenvironment profile, along with an increase in CD68+ and CD163 + cells and M1-like polarized macrophages ${ }^{6,8,9}$.

The evaluation of macrophage polarization on tissue sections is challenging. We published previously an immunohistochemical-based approach to evaluate this polarization ${ }^{10}$. This was based in experimental results, showing that STAT1 expression was proved in M1 macrophages in response to types I, II or III interferon, whereas its phosphorylated form (pSTAT1) binds to the promoter region of interferon-stimulated genes ${ }^{11-14}$; on the other hand, CMAF is expressed in M2 macrophages that produce interleukin (IL)-10, given that is an important transcription factor for IL-10 production ${ }^{15-17}$.

${ }^{1}$ Multidisciplinary Institute for Investigation in Pediatric Pathologies (IMIPP), CONICET-GCBA, Molecular Biology Laboratory, Pathology Division, Ricardo Gutiérrez Children's Hospital, Buenos Aires, Argentina. ${ }^{2}$ Institute for Pathology, Unfallkrankenhaus Berlin, Berlin, Germany. ${ }^{3}$ Oncology Division, Ricardo Gutiérrez Children's Hospital, Buenos Aires, Argentina. Correspondence and requests for materials should be addressed to P.C. (email: paola_ chabay@yahoo.com.ar) 


\begin{tabular}{|l|l|l|l|l|l|l|}
\hline \multirow{2}{*}{ Cell Subset } & \multicolumn{4}{l}{ Age group } & \multicolumn{2}{l|}{ EBV status } \\
\cline { 2 - 8 } & $\mathbf{2 1 0 y}$ & $\mathbf{7 1 0 y}$ & $\mathbf{p}$ & Positive & Negative & $\mathbf{p}$ \\
\hline Mean CD4+ cells $/ \mathrm{mm}^{2}$ & 766 & 733 & 0.08 & 771 & 750 & 0.67 \\
\hline Mean CD8+ cells $/ \mathrm{mm}^{2}$ & 773 & 572 & 0.089 & 707 & 592 & 0.26 \\
\hline Mean FOXP3 cells/mm ${ }^{2}$ & 1008 & 1459 & 0.017 & 1047 & 1405 & 0.32 \\
\hline Mean GrB+ cells $/ \mathrm{mm}^{2}$ & 613 & 556 & 0.3 & 528 & 736 & 0.91 \\
\hline Mean CD68+ pSTAT1+ cells $/ \mathrm{mm}^{2}$ & 247 & 130 & 0.021 & 215 & 178 & 0.69 \\
\hline Mean CD68+ CMAF+ cells $/ \mathrm{mm}^{2}$ & 20 & 69 & 0.09 & 33 & 49 & 0.29 \\
\hline Mean CD163+ pSTAT1+ cells $/ \mathrm{mm}^{2}$ & 165 & 125 & 0.56 & 153 & 147 & 0.66 \\
\hline Mean CD163+ CMAF+ cells $/ \mathrm{mm}^{2}$ & 67 & 121 & 0.017 & 81 & 102 & 0.08 \\
\hline
\end{tabular}

Table 1. Description of analyzed cell subsets according to age-group and EBV-association in pediatric classical Hodgkin lymphoma. GrB: Granzyme B. EBV: Epstein-Barr virus. Y: year. P-values from Mann-Whitney test.

We performed this study in order to study microenvironment composition in pediatric cHL, including an independent and geographically different series of cases from a single institution, in a region where EBV association is significant in patients under 10 years of age, and cHL subtype distribution is different from previous studies $^{18}$.

\section{Results}

Forty six pediatric $\mathrm{cHL}$ patients were included in this analysis. The mean age of the children was 9 years (3 to 16 years) and $76.1 \%(35 / 46)$ of cases were EBV-associated. Mixed cellularity (MC) was the most prevalent subtype (27/46 cases, $58.7 \%$ ), and was significantly associated to the $<10$ years age group ( $\mathrm{p}=0.0004$, Fisher exact test). Eighty percent $(28 / 35)$ of EBV-associated cases were in younger children ( $p=0.002$, Fisher's exact test). EBV presence was statistically related to the MC subtype (25/35 EBV+ cases, 71.4\%) over the nodular sclerosis (NS) subtype (7/35 EBV+ cases, $20 \%)(\mathrm{p}=0.0085$, Fisher exact test) (Table S1).

Comparable numbers of $\mathrm{CD} 4+, \mathrm{CD} 8+, \mathrm{FOXP} 3+$ and $\mathrm{GrB}+$ cells were observed at the tumor microenvironment (Table 1) in the 46 patients series. A statistically significant positive correlation was found only between FOXP3 + and GrB + cells ( $\mathrm{r}=0.30, \mathrm{p}=0.03$, Spearman correlation test) (Table S2). Young children showed a tendency of higher numbers of CD4+ and CD8 + cells $/ \mathrm{mm}^{2}(\mathrm{p}=0.08$ and $\mathrm{p}=0.089$, respectively, Mann-Whitney test) and were associated with lower FOXP3 + cells $/ \mathrm{mm}^{2}$ cell count $(\mathrm{p}=0.017$, Mann-Whitney test), in comparison with old children (Table 1). No significant differences were found among the cellular markers investigated between EBV + and EBV - cases ( $\mathrm{p}>0.05$, Mann Whitney test). Detailed description of $46 \mathrm{cHL}$ patients is provided in the Table 1 and Supplemental Tables.

CD68 + pSTAT1 + macrophages prevailed in relation to CD68+ CMAF+ macrophages (mean 197 cells $/ \mathrm{mm}^{2}$ and 47 cells $/ \mathrm{mm}^{2}$, respectively, $\mathrm{p}<0.0001$, Mann Whitney test), as well as CD163 + pSTAT1+ macrophages in relation to CD163+CMAF+ macrophages (mean 131 cells $/ \mathrm{mm}^{2}$ and 77 cells $/ \mathrm{mm}^{2}$, respectively, $\mathrm{p}=0.006$, Mann Whitney test) (Fig. 1). Predictably, a significant correlation was found between CD68+ pSTAT1+ and $\mathrm{CD} 163+\mathrm{pSTAT} 1+(\mathrm{p}<0.0001$, Spearman correlation test $)$, and between CD68 + CMaf + and CD163 $+\mathrm{CMaf}+$ cells $(p<0.0001$, Spearman correlation test). Young children exhibited increased numbers of CD68 + pSTAT1+ macrophages $/ \mathrm{mm}^{2}$ ( $\mathrm{p}=0.021$, Mann-Whitney test), while older children showed higher numbers of CD68+ $\mathrm{CMAF}+$ and CD163+CMAF + macrophages $/ \mathrm{mm}^{2}(\mathrm{p}=0.09$ and $\mathrm{p}=0.017$, respectively) (Table 1$)$. EBV presence did not showed any influence on macrophage composition (Table 1).

A positive correlation was proved between CD68+ pSTAT1 + macrophages and $\mathrm{GrB}+$ cells $(\mathrm{r}=0.46$, $\mathrm{p}=0.002$, Spearman's correlation), while CD68 + CMAF + macrophages exhibited an inverse correlation with CD8 + cells $(r=-0.49, \mathrm{p}=0.001$, Spearman's correlation). Regarding CD163, only an inverse correlation was found among CD163+ CMAF+ macrophages and CD8 + cells $(r=-0.32, p=0.049$, Spearman's correlation) (Table S2).

Regarding macrophage polarization and considering CD68 as macrophage marker, 76.9\% (30/39) of cases displayed M1-like polarization, 15.4\% (6/39) M2-like polarization and 7.7\% (3/39) comparable amount of M1and M2-like macrophages. When CD163 was used as macrophage marker, 54.5\% (18/33) of cases turned out to be M1-like polarized, 33.3\% (11/33) M2-like polarized and 12.1\% (4/33) exhibited equivalent numbers of M1- and M2-like macrophages.

M1-like polarization was statistically prevalent in patients younger than 10 years old, while, in contrast, M2 polarized cells presence were associated to older patients, considering both CD68 and CD163 as macrophage markers $(p=0.006$ and $p=0.0015$, respectively, Fisher exact test) (Table S3). Regarding EBV-association, no differences were observed when CD68 was considered as macrophage marker. However using CD163, EBV-associated cases displayed frequently M1-like polarization (66.7\% of cases, $16 / 24)$, while 66.7\% (6/9) of EBV-negative cases exhibited M2-like polarization ( $P=0.032$, Fisher's exact test) (Table S3).

Given that older children showed an immunoregulatory-rich tumor microenvironment, we evaluated if this characteristic could affect the macrophage composition. For this purpose, a ratio of FOXP3/GrB $>1.5$ as indicative of an immunoregulatory-rich microenvironment was defined, as published previously ${ }^{8}$. An immunoregulatory-rich tumor microenvironment was associated with lower mean of CD68+ pSTAT1 + cell numbers (mean 163 cells $/ \mathrm{mm}^{2}$ for FOXP3+/GrB ratio $>1.5$ vs. mean 270 cells $/ \mathrm{mm}^{2}$ for FOXP3 $/ / G r B$ ratio ratio $<1.5, \mathrm{p}=0.028$, Mann-Whitney test). In fact, M1-like polarization was the exclusive pattern observed in patients 


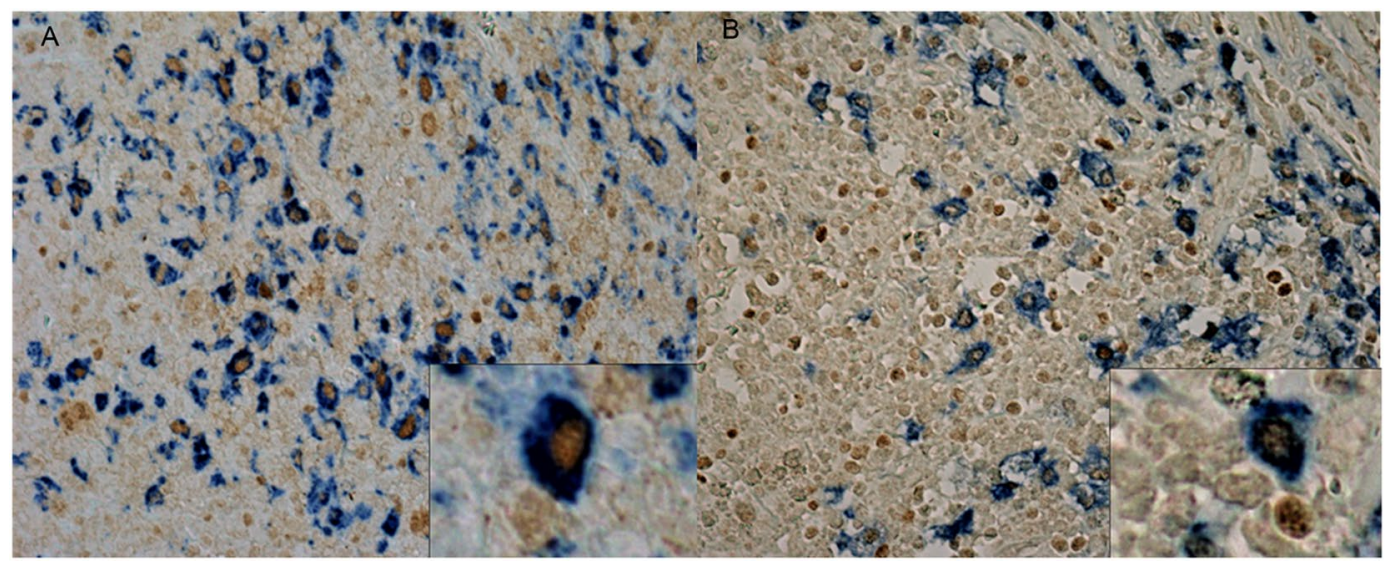

Figure 1. Representative double immunohistochemical staining undertaken on sections from cHL biopsies. Cytoplasmic CD68+ staining combined with nuclear pSTAT1+ macrophages (A). Cytoplasmic CD163+ staining combined with nuclear $\mathrm{CMAF}+$ macrophages $(B)$. Original magnification: $\times 400$.

with FOXP3/GrB ratio $<1.5$ defined by CD68 + pSTAT1 + approach. No difference was highlighted when CD163 was considered.

Finally, hierarchical cluster analysis was conducted to identify underlying patterns of tumor microenvironment cell subsets, in relation to both EBV status and age group (Fig. 2). In this analysis, 2 distinctive clusters were observed: cluster I included the EBV - negative cases, those with high M2-like macrophages cell count, as demonstrated by CD68 $+\mathrm{CMAF}+$ and $\mathrm{CD} 163+\mathrm{CMAF}+$ above the 50th percentile, and predominance of FOXP3 + over GrB + cells, given that the ratio Foxp3+: GrB $+>1.5$ prevails in this cluster. In contrast, the cluster II is mainly formed by EBV-associated cases and those with increased numbers of M1-like macrophages, confirmed by CD68+ pSTAT1 + and CD163+ pSTAT1+ cases above the 50th percentile, and predominance of $\mathrm{GrB}+$ over FOXP3 + cells, since the ratio GrB+: Foxp3+ is higher than 1.5.

\section{Discussion}

In pediatric cHL, only two studies were performed in children from developed socioeconomic populations ${ }^{5,7}$, while one group has extensively studied a pediatric population from a developing country, Brazil ${ }^{6,8,9}$. Gupta et al. ${ }^{5}$ and Englund et al. ${ }^{7}$ performed tumor microenvironment analysis in pediatric cHL cases from developed population (Canada and Sweden, respectively), in which cHL incidence, rate of EBV-association and subtype distribution is different. In developed countries, nodular sclerosis (NS) subtype is prevalent over mixed cellularity (MC). In fact, NS subtype was described in $91.6 \%$ of cases demonstrated by Gupta et al..$^{5}$, and in $69 \%$ of cases reported by Englund et al. ${ }^{7}$. In line with this, Barros et al. ${ }^{6,8,9}$ described in their pediatric series that $69 \%$ of cases were NS subtype, even though their study was performed in a developing population. In contrast, in our study $58.7 \%$ of cases were MC subtype. EBV was associated with $25 \%$ of pediatric cases from a developed population published Englund et al., particularly in patients younger than 10 years old ${ }^{7}$. Meanwhile $44.8 \%$ of cases were EBV-associated by Barros et al. in pediatric cHL cases from a developing country, without correlation with age-groups $s^{6,8,9}$. In contrast, we observed an increase in EBV-association in our series from a developing population along with a correlation with young age-group.

Barros et al. suggested a shift from a cytotoxic to a regulatory profile in the tumor microenvironment of pediatric $\mathrm{CHL}$ with the increase of the age ${ }^{8}$. In line with this, we observed a regulatory profile in children older than 10 years old, confirmed by a higher FOXP3 + cell counts. Nevertheless, an increment on the numbers of GrB + cells was not observed in younger patients, in spite of EBV prevalence on this specific group, which would be expected to trigger an antiviral cytotoxic profile. However it is remarkable that EBV-associated cases exhibited higher means of CD8 + cells $/ \mathrm{mm}^{2}$, as described previously ${ }^{8}$. The reasons why older children develop a tumor microenvironment rich in Treg cells, similar to adult $\operatorname{cases}^{19}$, are not clear yet. One possible explanation could be the increase of Treg cells numbers and suppressive function with the aging of the immune system ${ }^{20}$.

Polarized M1 macrophages are generally pro-inflammatory and anti-tumoral. In contrast, M2 macrophages were described as an immunoregulatory phenotype, which mediate tumor promotion ${ }^{21}$. In cHL, the immunomodulatory phenotype adopted by macrophages may be the potential mechanism by mean of which they promote immune evasion ${ }^{22}$. In this study we observed an increase in M1-like macrophages cell count and M1-like polarized tumor microenvironment in younger children. Also, this kind of polarization was linked to cytotoxic tissue signature, as evidenced by the increase in $\mathrm{GrB}+$ cells in M1-like polarized cases. In contrast, higher numbers of M2-like macrophages and M2-like polarized tumor microenvironment was observed in older patients and related to an immunoregulatory-rich environment, in line with previous results ${ }^{9}$. Together, our results support previous hypothesis that the tumor microenvironment in pediatric $\mathrm{cHL}$ is influenced by age, and a possible cross-talk between cells of adaptive and innate immune system in this microenvironment may contribute to the macrophage polarization ${ }^{6,8,9}$

In summary, a predominance of M1-like polarized macrophages in young children, as well as M2-like polarized macrophages and Treg cells in old children was observed, supporting the notion that pediatric cHL exhibits a particular tumor microenvironment composition when compared with adult cases ${ }^{6,8,9,23-25}$. 


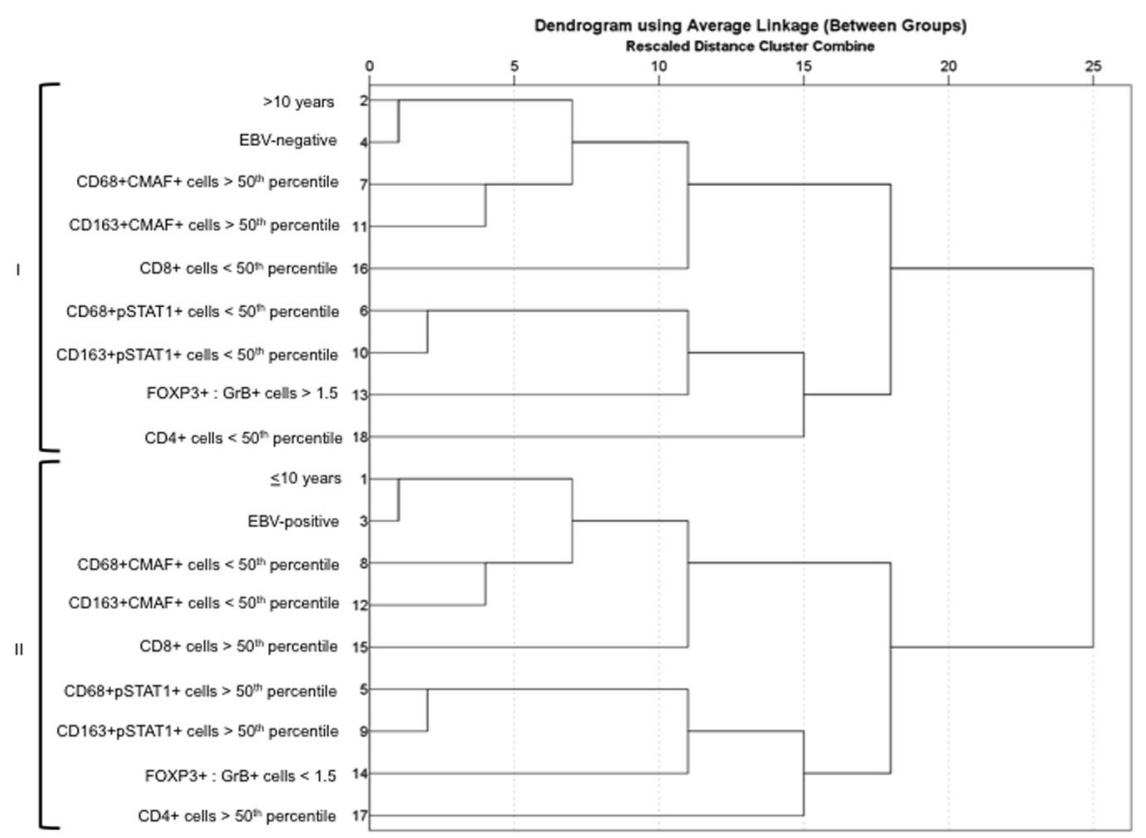

Figure 2. Dendrogram using average linkage obtained by hierarchical cluster analysis. Two main identified clusters (I and II) are indentified by brackets. Num, order of variable input. EBV: Epstein-Barr virus. GrB: Granzyme B.

\section{Methods}

Patients and samples. Formalin-fixed paraffin-embedded (FFPE) biopsy samples from 46 patients were collected retrospectively, on the basis of the availability of sufficient material, from the archives at Pathology Division, Ricardo Gutierrez Children's Hospital in Buenos Aires, Argentina, from 1990 through 2012, and a tissue microarray (TMA) was constructed as described ${ }^{26}$. From each case, two 3-mm-diameter cores, selected from two different representative tumor areas rich in HRS cells selected by the pathologists EDM and MHB, were included ${ }^{26}$.

The Ricardo Gutierrez Children Hospital Ethics Committee on Research (CEI) approved the study, and the subjects gave informed consent to the study. Diagnosis was revised according to the updated WHO scheme for lymphomas ${ }^{27}$. All methods were performed in accordance with the relevant guidelines and regulations.

Immunohistochemistry. Tissue Microarray (TMA) blocks including $46 \mathrm{cHL}$ cases were constructed, as previously described ${ }^{6,8,9}$. For each patient, two 1-mm-diameter cores, selected from two different representative tumor areas rich in Hodgkin and Reed-Sternberg cells, were included, as described ${ }^{6,8,9}$. All cases showed cores with representative tumor microenvironment. Immunohistochemistry (IHC) staining was performed on TMA sections with antibodies: CD4 (Leica, Newcastle, UK), CD8 (Dako, Gloustrup, Denmark), FOXP3 (Treg) (Abcam, Cambridge, UK), Granzyme B (GrB) (AbD Serotec, Oxford, UK). After incubation with primary antibody, immunodetection was performed using ZytoChem Plus HRP polymer kit (Zytomed Systems, Berlin, Germany), employing diaminobenzidine (DAB) chromogen as substrate, as described ${ }^{6,8,9}$. Sections were counterstained with hematoxylin.

In addition, macrophage polarization was assessed by double immunohistochemistry, as described previously $^{10}$. pSTAT1 or CMAF (Santa Cruz Biotechnology, Dallas, USA) were used as first primary antibodies and the detection of bound antibodies was performed using ZytoChem Plus HRP polymer kit (Zytomed Systems, Berlin, Germany), employing diaminobenzidine (DAB) as chromogen ${ }^{10}$. Then, CD68 (Dako) or CD163 (Novocastra, Wetzlar, Germany) antibodies were incubated as second antibodies, followed by detection with AP Polymer System (Zytomed Systems, Berlin, Germany), wiht Blue Alkaline Phosphatase substrate kit (Vector Laboratories, Burlingame, USA) as substrate. The sections were not counterstained ${ }^{10}$. Co-expression of pSTAT1 together with CD68 or CD163 was used to identify M1-polarized macrophages, while co-expression of CMAF in conjunction with CD68 or CD163, was performed to identify M2-polarized macrophages ${ }^{20}$. The ratio M1: M2 per case was assessed for each marker combination, and a polarized response (M1 > M2 or M2 > M1) was defined as one cell population $1.5 x$ higher than the other ${ }^{10}$.

Tumor microenvironment analyzes. For lymphocyte and macrophage markers evaluation, the slides were scanned with Aperio LV1 digital pathology slide scanner (Leica Biosystems, CA, USA) at a 200× magnification. Using Aperio Image Scope software (Leica Biosystems, CA, USA), each core was extracted as separated JPG file. After, the numbers of labeled lymphocytes were determined per $1 \mathrm{~mm}^{2}$ using the image analysis free software Image $\mathrm{J}^{28}$. The cells were counted optically without the use of plug-in. 
Epstein-barr virus association. Epstein-Barr virus association was evaluated by EBERs in situ hybridization (EBER-ISH) and immunohistochemistry. EBER-ISH was performed on FFPE tissue sections using fluorescein isothiocyanate (FITC)-conjugated EBERs oligonucleotides as probes, following the manufacturer's instructions (Dako). A monoclonal antibody anti-FITC labeled with alkaline phosphatase was used for the detection of EBERs probes (Dako), as described ${ }^{29}$.

Immunostaining was used to localize viral LMP1 expression in tumor cells, using monoclonal antibodies CS1-4 (Dako). IHC detection of primary antibody was carried out using a universal streptavidin-biotin complex-peroxidase detection system (UltraTek HRP Anti- Polyvalent Lab Pack, ScyTek, Logan, Utah, USA) according to the manufacturer's instructions ${ }^{29}$.

Statistical analysis. Statistical analysis was performed using GraphPad Prism 5 (GraphPad Software Inc, San Diego California USA). Children were divided in two age groups: young ( $\leq 10$ years) and old ( $>10$ years). Categorical variables were analyzed using Fisher's exact test or Chi Square test when necessary. The KolmogorovSmirnov test was used for testing normality. T-test or Mann-Whitney test was used to compare the means, when appropriate. Correlations between data were determined using Spearman or Pearson rank correlation index, when appropriate. All tests were two-tailed, and $\mathrm{p}<0.05$ was considered statistically significant.

To explore the structure of association among variables of tissue microenvironment, EBV presence and age-group, a hierarchical cluster analysis using average linkage and binary simple matching measure with Statistical Package for the Social Sciences 13.0 (SPSS) was performed.

\section{References}

1. Liu, Y., Sattarzadeh, A., Diepstra, A., Visser, L. \& van den Berg, A. The microenvironment in classical Hodgkin lymphoma: an actively shaped and essential tumor component. Semin Cancer Biol 24, 15-22 (2014).

2. Fowler, N. H. et al. Role of the tumor micro environment in mature B-cell lymphoid malignancies. Haematologica 101, 531-40 (2016).

3. Scott, D. W. \& Gascoyne, R. D. The tumour microenvironment in B cell lymphomas. Nat Rev Cancer 14, 517-34 (2014).

4. Catalano, V. et al. Tumor and its microenvironment: a synergistic interplay. Semin Cancer Biol 23, 522-32 (2013).

5. Gupta, S., Yeh, S., Chami, R., Punnett, A. \& Chung, C. The prognostic impact of tumour-associated macrophages and ReedSternberg cells in paediatric Hodgkin lymphoma. Eur J Cancer 49, 3255-61 (2013).

6. Barros, M. H., Hassan, R. \& Niedobitek, G. Tumor-associated macrophages in pediatric classical Hodgkin lymphoma: association with Epstein-Barr virus, lymphocyte subsets, and prognostic impact. Clin Cancer Res 18, 3762-71 (2012).

7. Englund, A. et al. The role of tumour-infiltrating eosinophils, mast cells and macrophages in Classical and Nodular Lymphocyte Predominant Hodgkin Lymphoma in children. Eur J Haematol 97, 430-438 (2016).

8. Barros, M. H., Vera-Lozada, G., Soares, F. A., Niedobitek, G. \& Hassan, R. Tumor microenvironment composition in pediatric classical Hodgkin lymphoma is modulated by age and Epstein-Barr virus infection. Int J Cancer 131, 1142-52 (2012).

9. Barros, M. H., Segges, P., Vera-Lozada, G., Hassan, R. \& Niedobitek, G. Macrophage polarization reflects T cell composition of tumor microenvironment in pediatric classical Hodgkin lymphoma and has impact on survival. PLoS One 10, e0124531 (2015).

10. Barros, M. H., Hauck, F., Dreyer, J. H., Kempkes, B. \& Niedobitek, G. Macrophage polarisation: an immunohistochemical approach for identifying M1 and M2 macrophages. PLoS One 8, e80908 (2013).

11. Katze, M. G., He, Y. \& Gale, M. Viruses and interferon: a fight for supremacy. Nat Rev Immunol. 2(9), 675-87. (2002).

12. Leopold Wager, C. M., Hole, C. R., Wozniak, K. L., Olszewski, M. A. \& Wormley, F. L. STAT1 Signaling Is Essential for Protection against Cryptococcus neoformans Infection in Mice. J Immunol. 193(8), 4060-71 (2014).

13. Murray, P. J. et al. Perspective Macrophage Activation and Polarization: Nomenclature and Experimental Guidelines. Immunity. 41(1), 14-20 (2014)

14. Zhang, Y. et al. Modular Analysis of Bioinformatics Demonstrates a Critical Role for NF- $\kappa B$ in Macrophage Activation. Inflammation. 37(4), 1240-53 (2014).

15. Saraiva, M. \& O'Garra, A. The regulation of IL-10 production by immune cells. Nat Rev Immunol. 10(3), 170-81 (2010).

16. Cao, S., Liu, J., Song, L. \& Ma, X. The protooncogene c-Maf is an essential transcription factor for IL-10 gene expression in macrophages. J Immunol. 174(6), 3484-92. (2005).

17. Van den Bosch, M. W. M., Palsson-Mcdermott, E., Johnson, D. S. \& O’Neill, L. A. J. LPS Induces the Degradation of Programmed Cell Death Protein 4 (PDCD4) to Release Twist2, Activating c-Maf Transcription to Promote Interleukin-10 Production. J Biol Chem. 289(33), 22980-90 (2014).

18. Chabay, P. A. et al. Pediatric Hodgkin lymphoma in 2 South American series: a distinctive epidemiologic pattern and lack of association of Epstein-Barr virus with clinical outcome. J Pediatr Hematol Oncol 30, 285-91 (2008).

19. Alvaro, T. et al. Outcome in Hodgkin's lymphoma can be predicted from the presence of accompanying cytotoxic and regulatory T cells. Clin Cancer Res 11, 1467-73 (2005).

20. Tulic, M. K. et al. Changes in thymic regulatory T-cell maturation from birth to puberty: differences in atopic children. J Allergy Clin Immunol 129, 199-206 (2012).

21. Andrew, D. Foey. Macrophages - Masters of Immune Activation, Suppression and Deviation, Immune Response Activation. Dr. Ht Duc (Ed.), In Tech (2014).

22. Tudor, C. S. et al. Macrophages and dendritic cells as actors in the immune reaction of classical Hodgkin lymphoma. PLoS One 9 , e114345 (2014).

23. Yang, L. \& Zhang, Y. Tumor-associated macrophages: from basic research to clinical application. J Hematol Oncol 10, 58 (2017).

24. Steidl, C., Connors, J. M. \& Gascoyne, R. D. Molecular pathogenesis of Hodgkin's lymphoma: increasing evidence of the importance of the microenvironment. J Clin Oncol 29, 1812-26 (2011).

25. Sánchez-Espiridión, B. et al. A molecular risk score based on 4 functional pathways for advanced classical Hodgkin lymphoma. Blood 116, e12-7 (2010).

26. Barros, M. H., Hassan, R. \& Niedobitek, G. Disease patterns in pediatric classical Hodgkin lymphoma: a report from a developing area in Brazil. Hematol Oncol 29, 190-5 (2011).

27. Swerdlow, S. H. et al. The 2016 revision of the World Health Organization classification of lymphoid neoplasms. Blood 127, 2375-90 (2016).

28. Rueden, C. T. et al. ImageJ2: ImageJ for the next generation of scientific image data. BMC Bioinformatics 18, 529 (2017).

29. Cohen, M. et al. Epstein-Barr virus presence in pediatric diffuse large B-cell lymphoma reveals a particular association and latency patterns: analysis of viral role in tumor microenvironment. Int J Cancer 132, 1572-80 (2013). 


\section{Acknowledgements}

This study was supported in part by grants from National Agency for Science and Technology Promotion (PICT 2014 No. 0478 - PID clinic No. 048). P.A.Ch., E.D.M. and M.V.P. are members of the CONICET Research Career Program.

\section{Author Contributions}

J.O. designed and performed research; B.M.H. and C.P. analyzed data; C.P. designed research; N.G. and D.M.E. performed immunostaining evaluation and classification of cases according to WHO; J.O., B.M.H. and C.P. made statistical analysis; J.O., B.M.H. and C.P. wrote the manuscript; P.M.V. corrected the manuscript; M.G.L. analyzed clinical data and survival.

\section{Additional Information}

Supplementary information accompanies this paper at https://doi.org/10.1038/s41598-019-49015-1.

Competing Interests: The authors declare no competing interests.

Publisher's note: Springer Nature remains neutral with regard to jurisdictional claims in published maps and institutional affiliations.

(i) Open Access This article is licensed under a Creative Commons Attribution 4.0 International License, which permits use, sharing, adaptation, distribution and reproduction in any medium or format, as long as you give appropriate credit to the original author(s) and the source, provide a link to the Creative Commons license, and indicate if changes were made. The images or other third party material in this article are included in the article's Creative Commons license, unless indicated otherwise in a credit line to the material. If material is not included in the article's Creative Commons license and your intended use is not permitted by statutory regulation or exceeds the permitted use, you will need to obtain permission directly from the copyright holder. To view a copy of this license, visit http://creativecommons.org/licenses/by/4.0/.

(C) The Author(s) 2019 